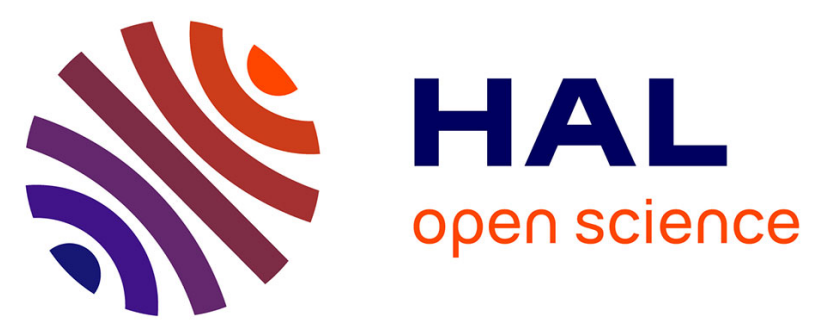

\title{
High-speed shadow imagery to characterize the size and velocity of the secondary droplets produced by drop impacts onto a heated surface
}

Guillaume Castanet, Pierre Dunand, Ophélie Caballina, Fabrice Lemoine

\section{To cite this version:}

Guillaume Castanet, Pierre Dunand, Ophélie Caballina, Fabrice Lemoine. High-speed shadow imagery to characterize the size and velocity of the secondary droplets produced by drop impacts onto a heated surface. Experiments in Fluids, 2013, 54 (3), pp.1489. 10.1007/s00348-013-1489-3 . hal-01430701

\section{HAL Id: hal-01430701 \\ https://hal.univ-lorraine.fr/hal-01430701}

Submitted on 30 Jul 2017

HAL is a multi-disciplinary open access archive for the deposit and dissemination of scientific research documents, whether they are published or not. The documents may come from teaching and research institutions in France or abroad, or from public or private research centers.
L'archive ouverte pluridisciplinaire HAL, est destinée au dépôt et à la diffusion de documents scientifiques de niveau recherche, publiés ou non, émanant des établissements d'enseignement et de recherche français ou étrangers, des laboratoires publics ou privés. 


\title{
High-speed shadow imagery to characterize the size and velocity of the secondary droplets produced by drop impacts onto a heated surface
}

\author{
G. Castanet $\cdot$ P. Dunand $\cdot$ O. Caballina $\cdot$ \\ F. Lemoine
}

\begin{abstract}
This study presents the developmental stages of an optical technique that aims at measuring simultaneously the droplet size and velocity. This technique is based on shadowgraphy associated with high-speed imaging. It is used to investigate the impacts of droplets onto a wall heated above the Leidenfrost temperature. Image analysis involves a detection of the droplet outlines for measuring their size and a tracking of the droplet trajectories to determine their velocities. This paper describes the different steps of the image processing. Solutions are also proposed to address the problems inherent to blurred, deformed, and overlapping droplets as well as the sizedependant effect of the depth of field. The technique is finally applied to several cases of rebound and splashing. Results show the many advantages resulting from the combination of size and velocity measurements.
\end{abstract}

\section{Introduction}

Measurement techniques, which are capable of characterizing droplets, are required in numerous investigations of two-phase flows, especially droplet/wall interactions. Quantities of interest are generally the size and velocity distributions and their related statistical moments, as well

G. Castanet $(\bowtie)$ P P. Dunand · O. Caballina · F. Lemoine UMR 7563, LEMTA, Université de Lorraine, ENSEM, 2 avenue de la Forêt de Haye, Vandœuvre-lès-Nancy, TSA 60604 - 54518 VANDOEUVRE CEDEX, France

e-mail: guillaume.castanet@univ-lorraine.fr

G. Castanet $\cdot$ P. Dunand - O. Caballina $\cdot$ F. Lemoine

UMR 7563, LEMTA, CNRS, ENSEM, 2 avenue de la Forêt de

Haye, Vandœuvre-lès-Nancy, TSA 60604 - 54518

VANDOEUVRE CEDEX, France as the droplet concentration. However, there are relatively few optical measurement techniques able to perform simultaneous non-intrusive measurements of the size and the velocity of individual particles. Phase Doppler anemometry (PDA) which is based on Doppler effect of the Mie scattering is certainly the most popular of these techniques. It has many advantages such as a high acquisition rate or the absence of calibration. However, one of its limitations concerns the reliability of its measurements in the presence of non-spherical droplets (Damaschke et al. 1998). Statistical confidence can be a problem if only a limited fraction of the droplets are spherical. Another difficulty is to determine local droplet number density and volume flux. Evaluating this flux implies knowing the cross-section of the measurement volume, but this quantity depends on many parameters such as the particle size, the flow direction of the particles, the optical configuration, and acquisition settings.

An alternative sizing technique is particle/droplet image analysis (PDIA). The improvement in the resolution and sensitivity of digital imaging systems has renewed the interest in these techniques. Backlight configuration for shadow imagery is generally preferred over direct imaging to visualize the outlines of irregularly shaped objects. The advantages are, for example, an accurate sizing of nonspherical and/or non-homogeneous particles, an easy optical alignment, and a relatively insensitivity to the optical properties of the particles (Lecuona et al. 2000). It is generally admitted that the accuracy of image processing techniques can be comparable with that of PDA (Blaisot and Yon 2005; Kashdan et al. 2003). However, PDIA has also some shortcomings. Its accuracy can strongly depend on the distance between the particle and the focal plane, as there is more ambiguity in defining the contour of unfocused droplets than focused ones. 
In many applications, a depth of field (DOF) criterion was therefore introduced to decide whether to count or not a particle given its degree of blur. However, choosing an appropriate and reliable DOF criterion is still an open question and quite different strategies have been attempted. Some authors based their criterion on a contrast value (difference in gray-level) between the particles and the background of the image (Kim and Kim 1994; Lebrun et al. 1996; Malot and Blaisot 2000). Other authors used the value of the gray-level gradient at the particle boundaries (Fantini et al. 1990; Lecuona et al. 2000). Koh et al. (2001) did a comparison between these two approaches. They showed that a gradient indicator is more appropriate for large droplets $(>30 \mu \mathrm{m})$, while a criterion based on a contrast value is more adapted to smaller droplets. However, the critical size of $30 \mu \mathrm{m}$ given by the authors is specific to their optical configuration and cannot be generalized.

If using a DOF criterion is unavoidable in most situations, it introduces some difficulties since the rejection of the blurred droplets is done without having eliminated all the biases, in particular the bias caused by the DOF dependency on the particle size. As the particle size becomes smaller, the DOF decreases drastically (Oberdier 1984), and hence, small particles are more likely to be miss-detected or rejected, resulting in a size distribution biased toward larger droplets. This problem can be very critical, if the detection of the particles relies on a fixed gray-level threshold, since it can be difficult to find a single threshold level fitting for all droplets. The number of the detected particles as well as their sizes would depend on the chosen threshold level. Several authors suggested therefore using a threshold based on a relative value, most frequently the half-height gray-level, i.e., the average of the levels at the center of the droplet and the background noise level (Hay et al. 1998; Kim et al. 1999; Kim and Kim 1994; Koh et al. 2001). Alternatively, gray-level gradient methods considered that the gray-level variation is the steepest at the particle boundaries. Nishino et al. (2000) obtained the particles outlines by fitting the maximum gradient points. Gradient-based identification methods were less used and their sensibility to DOF is less documented.

The aim of the present study is to develop a digital image analysis technique to investigate droplet size and velocity in the context of droplet/wall interaction. The focus will be placed in the film-boiling regime where a vapor layer is formed almost immediately below the droplet. This vapor film prevents the direct contact between the droplet and the hot wall. Although droplet/wall interactions received a considerable attention, these phenomena are still poorly understood. In particular, information is still missing concerning the droplet behavior: deformation, loss of momentum, heating, and vaporization. Advanced measurement methods are required to address these issues.
Among recent progresses, the droplet heating was characterized by Dunand et al. (2012a) and Castanet et al. (2009) using laser-induced fluorescence for measuring the droplet temperature. These latter measurements combined with the characterization of the heat flux at the wall surface allowed quantifying the droplet evaporation (Dunand et al. 2012b). Recently, the vapor film thickness was measured by Van der Veen et al. (2012) using an interference method and by Biance et al. (2003) in the case of sessile droplets with the help of a diffraction-based approach. The contribution of the present work concerns the droplet dynamic behavior, especially the size and velocity distributions of the outgoing droplets.

Many works have reported the use of a camera to observe the process of drop impact. Imaging systems were widely used to identify the impact regimes (rebound, splashing, deposition of a liquid film). Only in few studies, images were actually means of characterization of the droplet sizes and velocities. Recently Richter et al. (2005) and Müller et al. (2008) reported the use of shadow imagery to study droplet splashing. In these studies, shadow imagery was combined to particle image velocimetry (PIV) to obtain the Eulerian velocity field of the droplets as well as their size distributions. However, size and velocity measurements were disconnected. The limited spatial resolution of the camera was generally a problem for measuring the size of the smallest droplets with image analysis techniques, especially when studying drop splashing, because the size distribution of the secondary droplets can be rather broad. Cossali et al. (2005) developed a sizing method combining image analysis for the larger droplets and PDA for the smallest ones. The existence of a size range covered by the two methods allows normalizing their respective pdfs to form what was called by the authors an extended pdf. Like this, they were able to have size distributions from a few microns to millimeters. The same methodology was used later by Moreira et al. (2007) and Moita and Moreira (2012).

In the present study, a high-speed camera allows visualizing the impact of droplets by means of a shadow imaging system, and the time resolution of the visualization system is sufficient to undertake the tracking of the droplet trajectories. Image processing is divided into two distinct steps: identification of droplets and the tracking of their trajectories. In the paper, the emphasis is placed on the detection of the droplet shadow outlines and the separation of overlapping droplets. Then, a particularly robust tracking algorithm is presented. It is based on Multiple Hypothesis Tracking (MHT) methods, which are preferred techniques for particles tracking in cluttered environments (Chenouard et al. 2009). Finally, the performances of the measurement method are analyzed and its applicability and advantages for studying drop impact are illustrated. 


\section{Experimental setup}

The interaction between liquid sprays and hot solid walls occurs in a wide variety of industrial applications. For example, in direct injection engines, impacts of fuel droplets onto cylinder walls affect noticeably the combustion efficiency and the emissions of pollutants. Another application is related to spray cooling, which is widely used in the metal processing industry for its high efficiency, low coolant consumption, and the possibility to achieve a relative spatially homogeneous heat transfer (Bernardin and Mudawar 1997). When the wall is heated above the Leidenfrost temperature, a vapor layer is immediately formed, which prevents the direct contact between the liquid and the solid. This regime, generally called filmboiling regime, leads to a poor heat transfer between the liquid and solid. Hydrodynamics (related to the Weber number $W e=\rho V_{\mathrm{n}}^{2} d / \gamma$ where $d$ is the droplet diameter, $\rho$ the liquid density, $\gamma$ the surface tension and $V_{\mathrm{n}}$ the velocity normal to the wall) plays a significant role in the filmboiling regime (Wachters and Westerling 1966). For $W e<30$, water droplets spread and rebound. When $30<W e<80$, water droplets spread similarly as in the previous case, but separate into a number of satellite droplets after leaving the wall during their rebound. For $W e>80$, disintegration occurs in the initial spreading stage. This last regime is generally called splashing.

The experimental setup, presented in Fig. 1, is designed for investigating droplet impacts in the film-boiling regime. It was used in a previous study to determine heat transfers to the liquid in the film-boiling regime (Dunand et al. 2012a, b). A piezoelectric injector is used to produce a chain of mono-sized and equally spaced droplets by the disintegration of a cylindrical liquid jet. The breakup is driven by a Rayleigh-type instability that is triggered by the mechanical vibrations of the piezoceramic. For some specific frequencies of the vibrations, the liquid jet splits into equally spaced and monosized droplets. The size of the injector orifice and the inlet pressure allow adjusting separately the diameter $d$, the frequency $f$, and the velocity $V$ of the droplets. The droplet generator can be rotated to any prescribed angle $\alpha$ of incidence. Droplets collide with a thin slice of nickel heated by induction beyond the Leidenfrost temperature. The droplet generator can be rotated to modify the direction of the droplet stream and hence the normal velocity of the droplets and the impact regime. Details about the injection parameters used in the tested configurations of the present study are given in Table 1. The upper surface of the nickel on which the droplets are impacting is polished so as to obtain a very smooth surface. A high-speed (HS) camera (Phantom v710, Vision Research) is used to visualize the drop impacts. It can provide up to $7,500 \mathrm{fps}$ at full

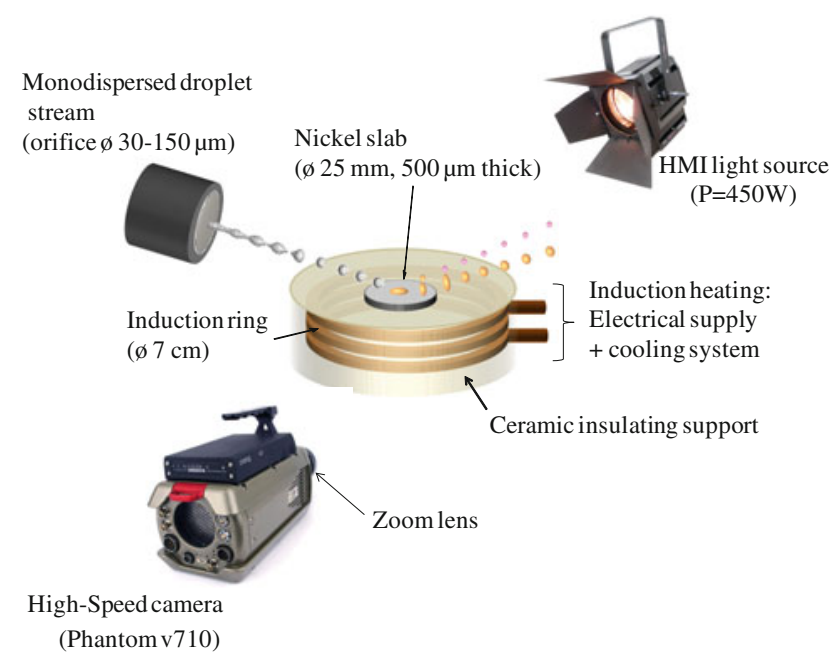

Fig. 1 Experimental setup

Table 1 Impact and acquisition parameters related to the cases in Fig. 2

\begin{tabular}{llll}
\hline & Case a & Case b & Case c \\
\hline$d(\mu \mathrm{m})$ & 115.9 & 171.4 & 118.3 \\
$V(\mathrm{~m} / \mathrm{s})$ & 8.71 & 8.59 & 8.47 \\
$\alpha\left(^{\circ}\right)$ & 19.8 & 57.3 & 50 \\
$V_{n}(\mathrm{~m} / \mathrm{s})$ & 2.98 & 7.23 & 6.49 \\
$W e_{n}$ & 20 & 117 & 69 \\
$f_{\text {inj }}(\mathrm{kHz})$ & 16.4 & 6.4 & 16.4 \\
$f p s(\mathrm{im} / \mathrm{s})$ & 84,000 & 70,000 & 72,000 \\
Resolution $(\mu \mathrm{m} / \mathrm{pxl})$ & 5.5 & 7.6 & 7.6 \\
Image size $(\mathrm{pxl})$ & $608 \times 120$ & $528 \times 144$ & $528 \times 144$ \\
\hline
\end{tabular}

resolution $(1,280 \times 800$ pixels, $20 \mu \mathrm{m}$ pixel size $)$. A zoom lens (OPTEM zoom $125^{\circ} \mathrm{C}$ minimized configuration) allows observing with a magnification ranging from 2.1 to 26. Given the small aperture of the zoom, it is required to illuminate the droplets from behind using a very bright light source (presently a $400 \mathrm{~W}$ HMI lamp with a parabolic reflector) in order to obtain contrasted shadow images with a short exposure time, set at $1 \mu \mathrm{s}$, its shortest possible value with the camera. Motion blur remains very limited given the moderate velocities of the secondary droplets in the studied conditions, this can be verified a posteriori by analyzing the velocity distribution of the outgoing droplets. A high magnification is necessary for accurately measuring the droplet size but in the same time, time-resolved visualizations require having rather high acquisition rates, typically acquisition rates are close to $80,000 \mathrm{fps}$ when looking at a splashing. This rate can be only achieved by a significant reduction of the number of pixels. The magnification of the zoom lens is adjusted in order to visualize the whole impact process and its values for the cases presented in this study 
(a)

(b)
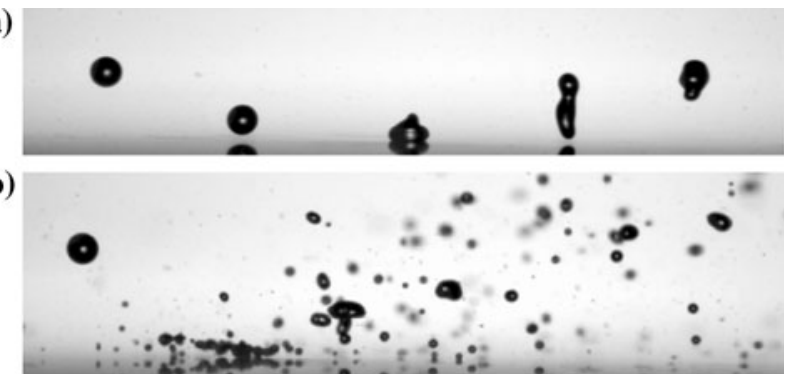

(c)

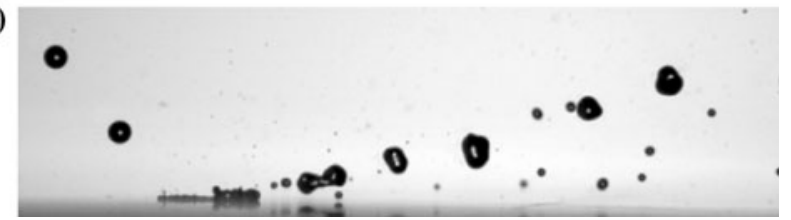

Fig. 2 Examples of rebound and splashing of water droplets in the film-boiling regime at wall temperature of $650{ }^{\circ} \mathrm{C}$

are given in Table 1. In practice, it was possible to reach a spatial resolution up to $5 \mu \mathrm{m}$ per pixel which is acceptable for drop sizing, while the number of pixels remains high enough to observe the whole impact and perform particle tracking velocimetry at the same time.

Figure 2 shows typical images of rebound and splashing obtained with this optical configuration. The frame rate and the pixel resolution corresponding to these acquisitions are indicated in Table 1 as well as the injection parameters. These three cases are extracted from a larger set of experiments, and they have been chosen to evaluate the potential of the developed image analysis.

\section{Identification of droplets and size measurements}

All the computations performed for the processing of the images have been done on a standard personal computer using Matlab and its image processing toolbox.

\subsection{Image pre-processing}

The processing begins with a normalization of the images. This stage aims at obtaining a uniform background in the image, given that the light source can be non-homogenous. The normalization corresponds to a division of the images by a background. The procedure is similar to that proposed by Blaisot and Yon (2005). The normalized image $I_{\text {norm }}$ is computed using:

$I_{\text {norm }}(i, j)=\beta \frac{I(i, j)-I_{0}(i, j)}{I_{b}(i, j)-I_{0}(i, j)}$,

where $I(i, j)$ is the gray level of pixel $(i, j)$ in the image $I, I_{b}(i, j)$ is the gray level of the background image (obtained without any object), and $I_{0}(i, j)$ corresponds to the noise level of the grabbing setup (i.e., the dark reference image obtained with the objective closed). $\beta$ is a correction factor introduced to account for the fact that the light source may be not temporally stable which leads to a difference in the global intensity level between the backgrounds of images $I_{b}$ and $I$. The parameter $\beta$ is adjusted so as to minimize the difference in background. Given the shortness of the acquisition time, the background of the images is not changing in a sequence of frames and $\beta$ takes a unique value for the processing. After normalization using Eq. (1), the objects are more easily identified (Fig. 3a). Normalized images are also corrected from the barrel distortion. A grid of periodically spaced dots is used for this correction. A spatial transformation is inferred from the coordinates of the dot centers, and the magnification factor is deduced knowing the distance between the dots of the grid. The barrel distortion does not affect the points near the center

(a)

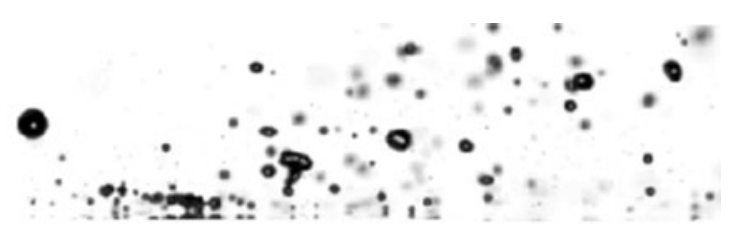

(b)

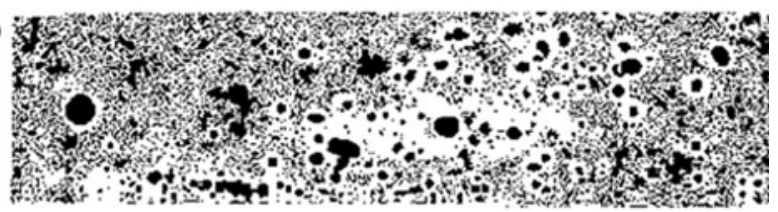

(c)

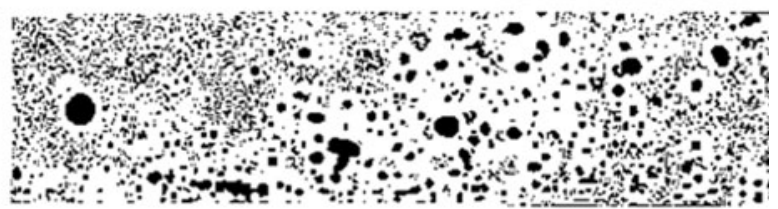

(d)

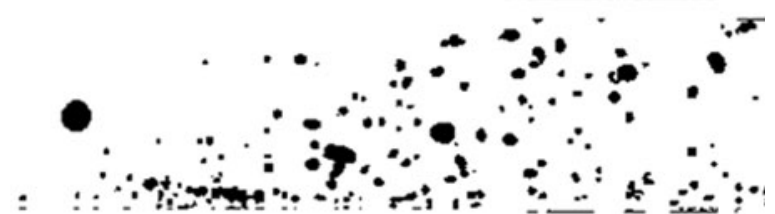

(e)

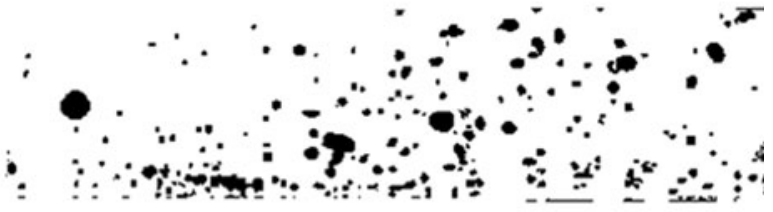

(f)

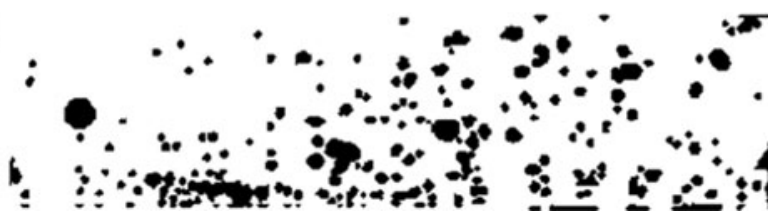

Fig. 3 Illustrations of the particle detection method in the case of the splashing in Fig. 2b. a Normalized image, b regions where $L<0$, c regions where $L<0$ after Gaussian filtering, $\mathbf{d}$ regions where $L<0$ and minimum $L_{\min }<L_{1}$ after Gaussian filtering, e regions where $L<0$ and $L_{\min }<L_{1}$ after Gaussian filtering $\left(L_{1}\right.$ is greater than in case d), $\mathbf{f}$ selected regions for contour reconstruction based on $\mathbf{e}$ ) 
of the image. As the grid is regular, it is then possible to estimate what would be the position of any of its dots if there was no distortion. Denoting $\left(x_{i}, y_{i}\right)$ the coordinates of the $i$ th dot centers on the image of the grid and $\left(u_{i}, v_{i}\right)$ its coordinates without distortion,

$\left[\begin{array}{ll}u_{i} & v_{i}\end{array}\right]=\left[\begin{array}{llllllllll}1 & x_{i} & y_{i} & x_{i} y_{i} & x_{i}^{2} & y_{i}^{2} & x_{i}^{2} y_{i} & x_{i} y_{i}^{2} & x_{i}^{3} & y_{i}^{3}\end{array}\right] \cdot T$,

where the matrix $T$ has a size 10-by-2. The elements of $T$ are determined using the least mean square method. Finally, the third-order polynomial transformation defined by $T$ in Eq. (2) is applied to all the pixels of the image.

\subsection{Detection of the droplet outlines}

Secondary droplets can be produced with a wide distribution of sizes. Detecting the smallest droplets is of prime importance given their large occurrence. Segmentation methods based on predefined values of gray-level or gradient thresholds are not well adapted to this situation since it is extremely difficult to find a suitable threshold value regardless the droplet size. Difficulties are also related to the presence of blurred droplets that can modify locally the background around the in-focused droplets. Separation of neighbor droplets, when they are very close to each other, can be very difficult if a low threshold value is chosen. In this study, these considerations led to take the steepest slope as an indicator of the droplet outlines. The developed approach is adapted from the general method of the Laplacian of the Gaussian (LoG) used in particular for blob structure detection (Acharya and Ray 2005).

Particle detection consists in searching the zeros of the Laplacian of the normalized images. The interior of the droplets corresponds then to the zones where the Laplacian is negative. As it can be seen in Fig. 3b, normalized images are too noisy for using directly their Laplacian $L$. To reduce the noise, a low-pass filter (for instance, a Gaussian filter, a circular averaging filter, or a Wiener filter) can be applied before calculating the Laplacian. However, this filtering should remain moderate in order not to enlarge the droplets or to risk to make the smallest droplets disappear. After Gaussian filtering, ripples (fluctuations inherent to the calculation of the second derivative in the Laplacian) are still visible, but they are less numerous (Fig. 3c). As ripples and noise can lead to the detection of false particles, a threshold is applied on the image $L$ of the Laplacian. Valid particles are defined as negative regions of $L$ (which are normally the droplets) that contain at least one pixel where the Laplacian value verifies the condition $L<L_{1}<0$. In other words, the minimum $L_{\min }$ of the Laplacian in each of these negative regions has to be smaller than a threshold value $L_{1}$. The value of $L_{1}$ is set to slightly exceed the height of the remaining ripples. Figure $3 d$ shows the zones, where the Laplacian is negative (like in Fig. 3c) and where the minimum $L_{\min }$ satisfies the thresholding $L_{\min }<L_{1}$.

In practice, it has been possible to retain only valid droplets with this method. Large and unfocussed objects are the first to be eliminated when increasing the value of $L_{1}$. Figure $3 \mathrm{e}$ is obtained with a lower value of $L_{1}$ than in Fig. 3d, and it is apparent that more unfocussed particles are present in that image. In Fig. 3e, small objects that are hardly visible in the original images (Fig. 3a) become apparent. It has been carefully checked that these objects were really droplets by observing that they really move in the frame series following ballistic trajectories. Capturing these very small droplets having a poor contrast was one of the main challenges of the detection.

Finally, the contour function of Matlab is used to find the closed lines corresponding to $L=0$ (Fig. 4), which can be interpreted as the edges of the droplets. Interpolation by the contour function allows having subpixel contour extraction. To reduce the computation time, the research of the droplet outlines is performed only in regions of interest (ROI) encompassing each zone where $L<0$. Figure $3 f$ shows the ROI resulting from Fig. 3e.

The detection method is well adapted to identify large and small droplets, whose contrast is very different (Fig. 4a). However, there is obviously a compromise between the value of $L_{1}$ and the strength of the low-pass filter. Strengthening the filtering allows working with lower values of $L_{1}$ but at the same time an artificial increase in the droplet size is at risk. Alternatively, contours can be extracted from the Laplacian of a less filtered image, while the ROI remain unchanged. This method proved to be very effective to capture all the droplets.

Presently, rotationally symmetric Gaussian low-pass filters of size 3 pixels were used for the selection of the ROI and the extraction of the contours. The standard deviation was fixed at 0.75 for the selection of the ROI and 0.3 for the extraction of the contours. It was not necessary to change the value of $L_{1}$ (about $-10^{-2}$ ) and the radial extend and the standard deviation of the Gaussian filter for all the tested conditions, as these parameters prove to be rather insensitive to the background level and the presence of blurred droplets (Fig. 4b). We also verified that similar results can be obtained using a circular averaging filter or a Wiener filter.

\subsection{Separation of overlapped particles}

Unfocused droplets can overlap more or less completely the focused particles and then have a negative influence on the measurements. In cluttered environments, many droplets have trajectories that intersect one another. A separation method that would correct for all the possibilities is 
(a)

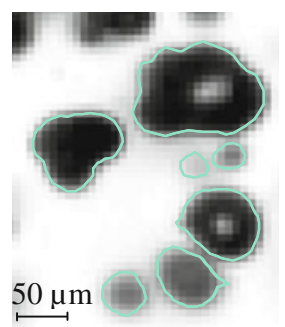

(c)

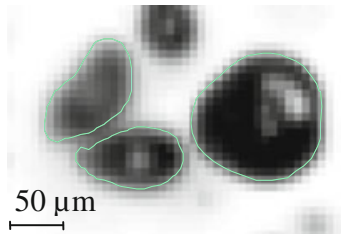

(e)

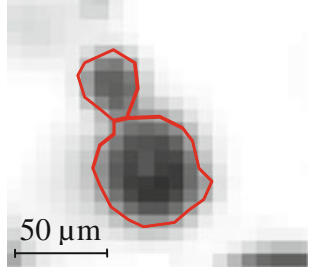

(g)

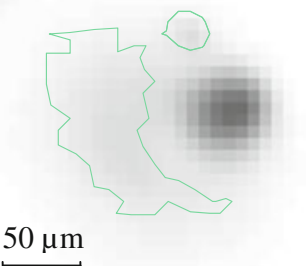

(b)

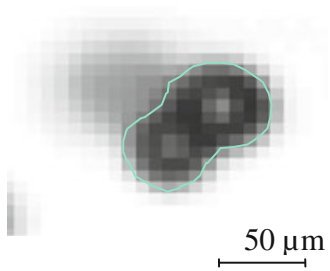

(d)

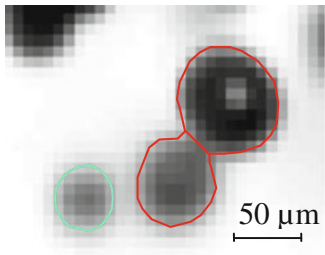

(f)

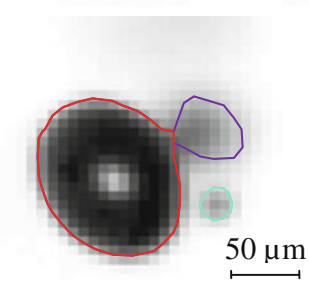

(h)

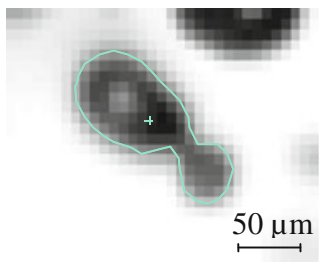

Fig. 4 Reconstruction of the particle contours in several ROI (in green: contour lines, in red: contours lines after separation of overlapping valid objects, in violet: contour lines after separation of overlapping invalid objects)

almost impossible. The use of parameters such as the object circularity and convexity to detect objects made of overlapped particles (Lecuona et al. 2000) is not really relevant in the present case, since the droplets can be strongly deformed after wall collision and breakup. In practice, the previously described detection method allows separating most of the droplets when they are partially overlapping from the very beginning of their identification (Fig. 4c). If a separation is undertaken, it is mainly for the tracking of the particle trajectories since missing particles can create severe difficulties.

Two strategies of separation are used in parallel. In the first one, the gray-level image is seen as a topographic relief where droplets are mountains. The watershed transform (Beucher and Meyer 1993) allows finding valleys, which are of interest for separation of clustered droplets. The height of the cervix (highest point in a given valley) is compared to the heights of the closest peaks to decide

whether or not to separate along the valley line. An example is given in Fig. 4d.

A second method analyses the curvature of the object outlines and identifies pairs of points that correspond to a narrowing of the contour. The object is cut straight if the distance between the pair of points is small enough (typically 2 pixels) (Fig. 4e).

The two methods have some complementarities. In practice, conditions to apply the second one are more frequent. After separation, newly formed objects can be eliminated if the Laplacian of their image does not reach the threshold $L_{1}$ (Fig. 4f).

Despite these approaches, some cases remain problematic, for example, finding the outline of a blurred droplet behind a focused one (the obtained contour is partial as it can be seen in Fig. 4g). In other cases, it can be difficult to decide whether a separation is effectively needed (Fig. 4h).

\subsection{Computation of droplet parameters}

The diameter $d$ of a droplet is based on the area $S_{d}$ inside the sub-pixel contour. Assuming a circular shape, $d$ is given by $\sqrt{4 S_{d} / \pi}$. For all the particles, the vertical and horizontal lengths of the particle (dimensions of the bounding box) are also extracted. The centroid of the particle is calculated from all points of the area $S_{d}$ inside the contour. It is interpreted as the center of mass of the particle for its tracking. Depth of field (DOF) can potentially introduce biases in the size measurements. The graylevel gradient at the particle boundaries $G$ and the maximum gray-level intensity $I_{\max }$ inside the particle are computed as they have been suggested as a relevant indicator for the DOF. The maximum gray-level intensity has been extensively used in previous works to calculate the contrast coefficient (Blaisot and Yon 2005; Lee and Kim 2004). In the case of rebounds, the spreading diameter is of particular interest. For droplets in apparent contact with the wall, the contact line, defined by the intersection between the droplet outline and the wall surface, is also extracted and the spreading diameter is determined.

\section{Tracking of the particles trajectories}

Particle tracking velocimetry (PTV) is possible because of the large acquisition rate of the high-speed camera at a pixel resolution compatible with particle sizing. PTV was chosen from scratch over particle image velocimetry (PIV) as it is at risk that droplets could have very different displacements within an interrogation window especially near the breakup zone at the wall collision. Moreover, PIV allow obtaining the Eulerian velocity field of the droplets, 
while a tracking of the particle trajectory would result in a Lagrangian description of the flow, particularly valuable for the modeling of the droplet-wall collisions. In turn, the tracking approach enables to correct the bias of the size distribution toward the slowest droplets. Without tracking, the slowest droplets that appear in more images than the fastest ones are counted more times.

\subsection{Tracking algorithm}

The algorithm is based on a Multiple Hypothesis Tracking (MHT) method. This kind of tracking method can be easily adapted to the case of droplet splashing. It is a preferred technique for solving the problem of data association in modern multiple targets tracking systems in cluttered environment (Chenouard et al. 2009; Reid 1979). The basic approach is to generate a set of data-association hypothesis to account for all possible origins of every measurement (here the positions of the centroids of the detected particles). In practice, the method relies on building all the possible associations between tracks and measurements for a number of successive frames and comparing them. All the hypothetical associations can be represented as a tree, whose branches correspond to a potential track. Algorithm is divided into several stages: initiation of tracks, track formation and extension, track termination, clustering, and selection of the potential tracks.

\subsubsection{Initiation of tracks}

Each object in a frame starts a new branch or track.

\subsubsection{Track formation and extension}

A branch existing up to frame $i$ is extended to the next frame by association with an object in frame $i+1$. Each branch at frame $i$ can thus generate a vast number of new branches at frame $i+1$. To prevent having a huge number of new branches, only associations, that have the higher probability, are considered in practice. Before a new hypothesis is created, the candidate target must satisfy a set of conditions. If the branch contains more than two elements, it is possible to predict the position of a candidate target based on the last known displacement in the track of this branch (acceleration/deceleration is accounted for in case of 3 elements and more in the branch). The candidate target must lie into a circle of confidence around the anticipated position to insure the continuity of the trajectories of the tracks. Conditions therefore relate to the direction and the length of the velocity vector.

In a cluttered environment (like in a splashing), a droplet that is visible in a given frame can disappear temporary for a certain number of frames if it is hidden by another droplet. A droplet can also disappear definitively if it breaks up, coalesces with another droplet,or comes out of the field of view. To handle the case of a temporary disappearance, it is accepted that a branch cannot be extended at the frame $i+1$. The possibility of an extension for this branch will be considered again with objects in frames $i+2, i+3$, etc... Delaying the decision of extension can increase considerably the number of branches to examine and the risk of errors in the tracking. In practice, this delay is limited to one or two frames. Unfocussed objects are considered for possible associations, as they can be part of a trajectory. DOF criterion presented later is for eliminating tracks that do not contain any object in focus.

\subsubsection{Track termination}

If a branch has not been extended for a certain number of frames (typically for 2 or 3 frames), the branch is terminated and its extension will not be examined any more.

\subsubsection{Clustering and selection of the potential tracks}

Branches that have been completed in the previous stage are considered as potential tracks if they contain a sufficient number of elements (typically at least six or seven elements), otherwise they are eliminated because their probability is assumed to be too low. This rule of selection implies that the frame rate of the high-speed camera is high enough so as to capture any droplet a sufficient number of times in the frame sequence. The retained branches are gathered in clusters when they have an object in common. Several reasons can explain that two branches could intersect each other. Two particles can overlap almost completely to the extent that they could not be separated with the methods described in Sect. 3.3. Another possibility is that one of the branches corresponds to an erroneous tracking. For example, an error can occur when two particles are located very closely. One of them can be incorrectly attributed to a branch in place of the other. In each cluster of intersecting branches, a selection is made so as to retain the branches that are the more probable. A branch corresponding to the most regular trajectory (i.e., having less change in velocity direction) is formed at first. The diameter is not used here as it may fluctuate due to droplet shape oscillations (Fig. 14). The objects excluded from that first branch are grouped together to see whether they can form another potential track with the required number of elements, and so on. By that process, an object belonging to several branches is attributed to a single branch (the one where it has the most regular connections) and in the other branches a hole is made.

After analysis of all clusters, it is considered that only valid tracks remain. Figure 5 gives an example of tracks 


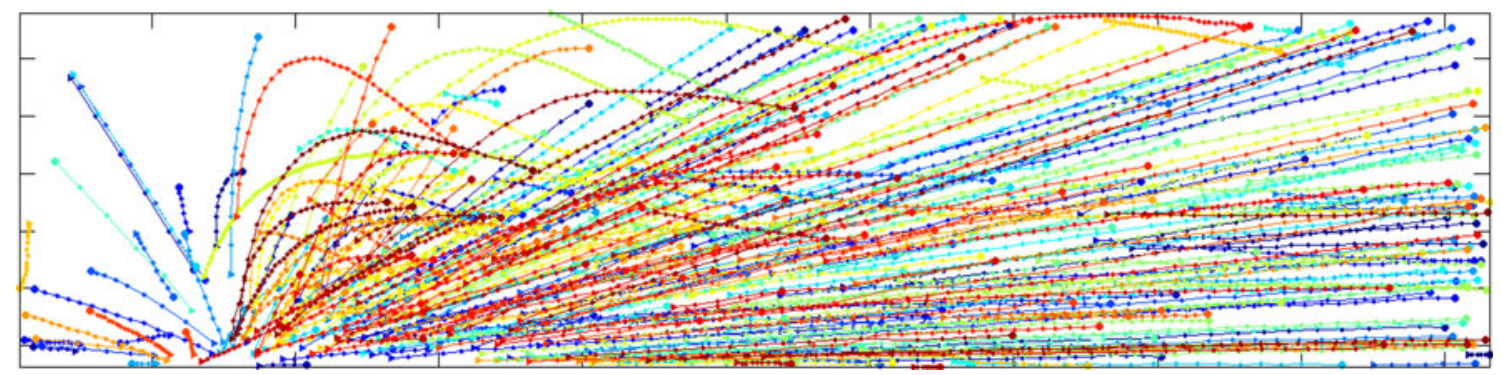

Fig. 5 Examples of tracks completed in the time interval of 30 frames in the case of the splashing in Fig. $2 b$ about 350 tracks are completed (random colormap)

obtained in the case of the splashing of Fig. 2b. For the sake of clarity, only few tracks completed over a short number of frames ( 30 frames) are featured in this figure.

\subsection{Droplet classification}

The sign of the velocity is used to sort the droplets into classes. Tracks with an initial velocity directed upward are classified as secondary droplets. A heuristic-based approach allows distinguishing the primary droplets from the ternary droplets (secondary droplets coming back to the wall with a downward velocity). The basic idea is that ternary droplets are smaller in size and have a lower downward velocity. An illustration of the classification is given in Fig. 6, where the spatial distribution of the droplets in frequency is plotted. The frequency values reported in the color scales of Fig. 6 correspond to the number of particles detected in areas of 10 by 10 pixels divided by the duration of the acquisition (about $57 \mathrm{~ms}, 4,000$ images). For the primary droplets in Fig. $6 \mathrm{~b}$, the measured frequency is about equal to that of the supply voltage of the piezoelectric injector, i.e., $6.4 \mathrm{kHz}$. This indicates that none of these droplets has been missed by the detection and tracking procedures.

For the secondary droplets, the frequency decreases with the distance from the impact, as droplets eventually leave the DOF (Fig. 6c). Figure 6e shows the frequency of droplets that are not included in a track. These droplets are mainly located near the impact region. In the case of a splashing, droplets in contact with the wall are excluded from the tracking as no relationship is sought between primary and secondary droplets. In the case of a rebound (with or without formation of satellite droplets), droplets in contact with the wall are retained in the tracking, as it is relatively simple to connect every primary droplet to its subsequent droplets in contact and their outcoming secondary droplets.

\section{Size measurement accuracy and the effect of the depth of field}

A transparent glass target dotted with discs of different sizes is used to assess the accuracy of the size (a)

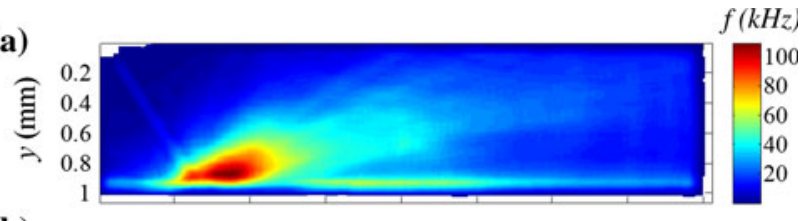

(b)

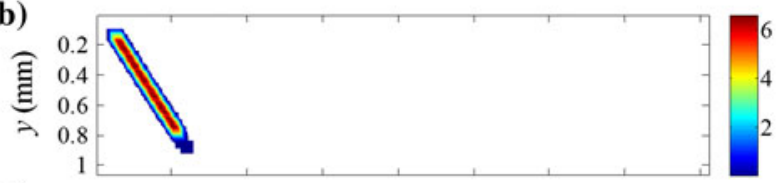

(c)

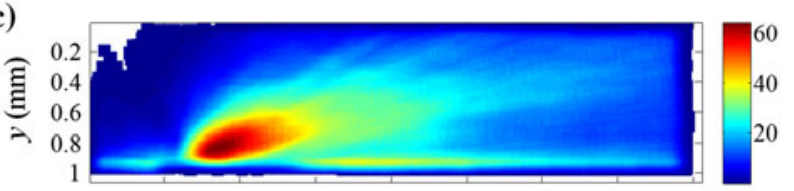

(d)

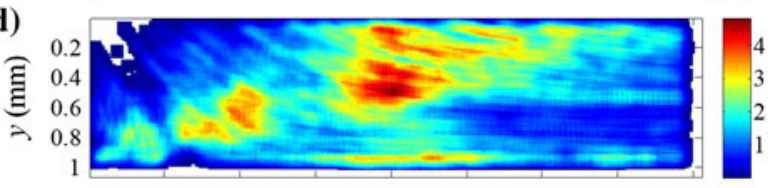

(e)

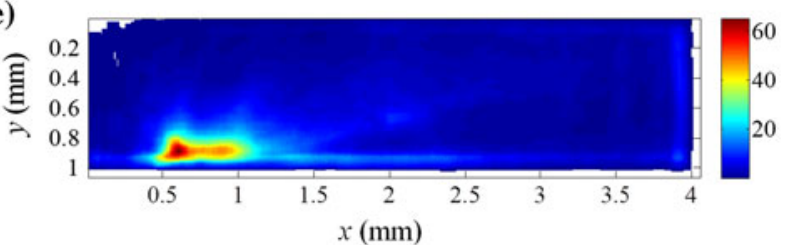

Fig. 6 Spatial distribution in frequency of the droplets by categories in the case of example in Fig. $2 b$ (a all the detected objects, $\mathbf{b}$ primary droplets, c secondary droplets, d ternary droplets, e objects not included in a track)

measurements and to characterize the effect of the DOF (Fig. 7). Disc diameters range from 4 to $500 \mu \mathrm{m}$, which covers the whole range of droplet sizes encountered in the experiments. The same parameters are utilized for the detection of the patterns as in the study of the droplet/wall interactions.

Figure 8 presents the sizing errors as a function of the actual disc sizes when the plate is located at the focal plane of the camera for the two illumination levels displayed in Fig. 7. Measurements are performed with the same magnification as indicated in Fig. 2b, c, i.e., a spatial resolution of $7.6 \mu \mathrm{m}$ per pixel. A systematic error of about $4 \mu \mathrm{m}$ is 
(a)

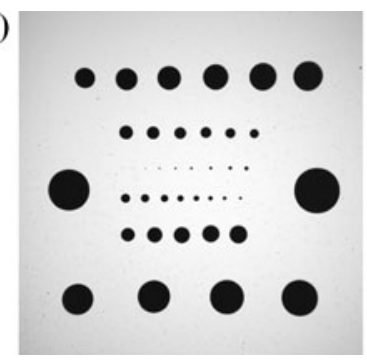

(b)

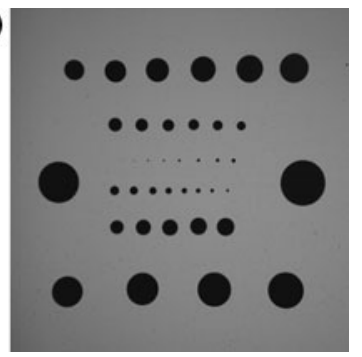

Fig. 7 Calibration plate dotted with discs ranging from 4 to $0.5 \mathrm{~mm}$. The two illuminations tested

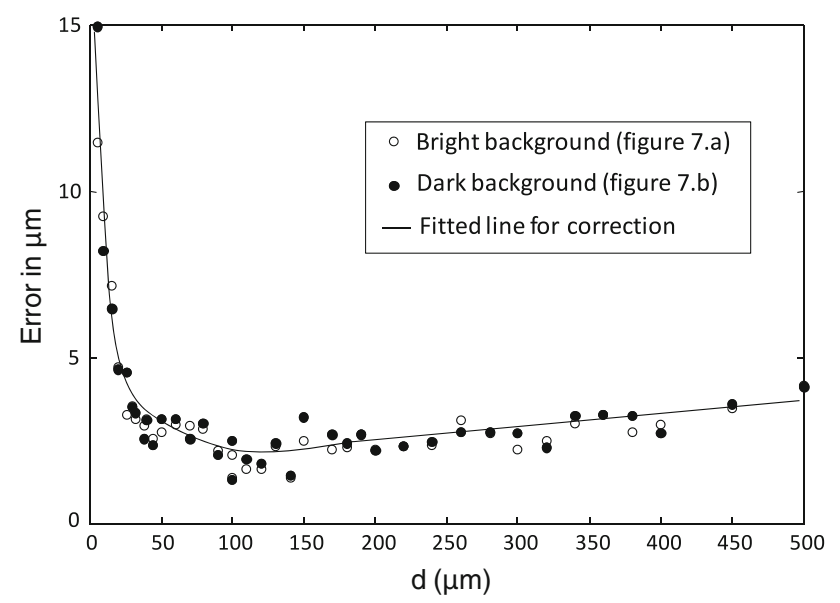

Fig. 8 Errors in size measurement as a function of the disc diameters

observed for droplets larger than $40 \mu \mathrm{m}$ whatever the background level. For the smallest droplets, the overestimation of the sizes is more important and the sensitivity of the drop sizing to the illumination is also very weak. Errors observed in Fig. 8 are mainly due to the pixel resolution. Subpixel interpolation of the contour is clearly less accurate for very small objects. Additionally, the low-pass filtering and the diffraction can also contribute to an increase in the apparent size of an object, when it is small. In the following, the fitted curve in Fig. 8 is used to correct these different sources of error. For that, the sizing error quantified with the glass target in Fig. 8 is subtracted from the apparent size of the objects.

The DOF was also characterized as droplets produced by a splashing can move away from the plane formed by the incident droplet stream and the normal to the wall. The glass target is moved back and forth along the optical axis of the camera. DOF is defined as the maximum distance over which a droplet can be detected and processed. As expected, the DOF increases with the size of the object (Fig. 9). It can be noticed that a disc of $10 \mu \mathrm{m}$ (roughly the smaller size detectable in practice with the system) is visible on a thickness of about $1 \mathrm{~mm}$. The depth of the

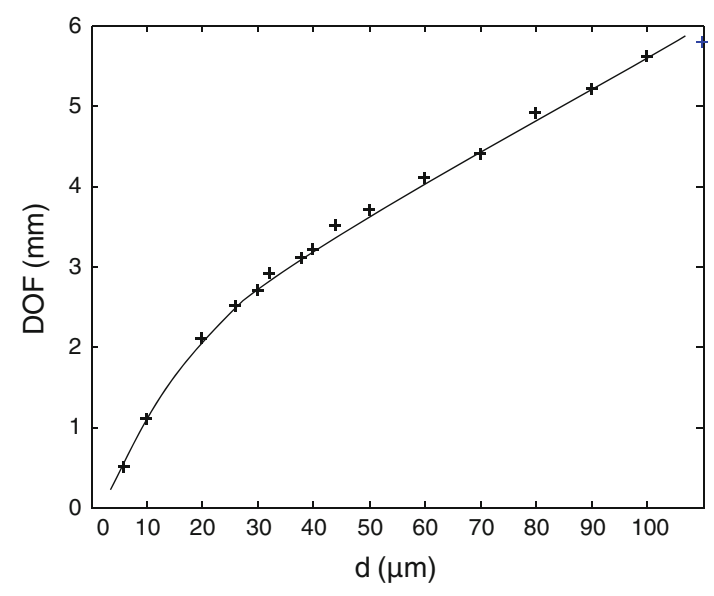

Fig. 9 DOF versus sizes of the discs on the calibration plate

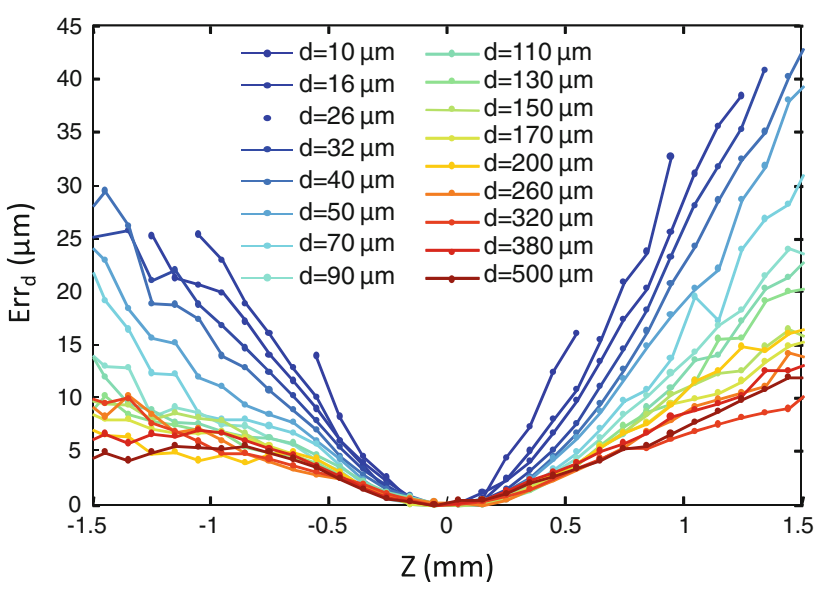

Fig. 10 Size measurement errors as a function of the distance from the focal plane

measurement volume increases sharply with the disc size: it is $2 \mathrm{~mm}$ for the disc of $20 \mu \mathrm{m}$. DOF appears sufficient to visualize most of the secondary droplets produced in the experiments.

Figure 10 shows the measurement error $\operatorname{Err}_{d}$ in size as a function of the distance $z$ from the focal plane. In this figure, $\operatorname{Err}_{d}$ corresponds to the difference between the measured size and the actual size of the discs. The systematic error in Fig. 8 has been subtracted to the measured size. $\operatorname{Err}_{d}$ is thus equal to 0 at the focal plane $(\mathrm{z}=0)$. As defined, $\operatorname{Err}_{d}$ is related to the defocusing error. Errors due the defocusing are moderate in an interval $z= \pm 1 \mathrm{~mm}$, which corresponds to the region where most of the droplets are located in the experiments (Fig. 18). The size of an unfocused droplet is always overestimated when its boundary is defined as the steepest gradient.

In Fig. 10, a dissymmetry between the foreground and the background can be observed. This dissymmetry is negligible in the interval $z=-1 \mathrm{~mm}$ to $z=+1 \mathrm{~mm}$. Few attempts have been reported to model this kind of 
asymmetry. Bongiovanni et al. (1997) are certainly the only ones that were able to capture it but in the case of bubbles. They used a ray tracing program and incorporated the complete arrangement of their optical setup in the computations. Using geometrical optics, they missed the effect of diffraction. Fdida et al. (2010) reported the same kind of asymmetry. They attributed it to a 'perspective effect' and showed that it can be circumvented using a telecentric objective. To account for that effect, the authors suggested modifying the radial extend of the Point Spread Function (PSF). The evolution of the radial extend of the PSF as a function of the position relative to the focal plane is obtained by an experimental calibration very similar to that presented in the present study. Nevertheless, as it is not possible in practice to know whether an out-of-focused droplet is located in the foreground or in the background toward the focal plane, the simple and the best correction is probably an average correction, half-way between the corrections fitted for $z<0$ and $z>0$. The sizing error is thus described by a polynomial of order 2 :

$\operatorname{Err}_{d}=A(d) z^{2}$,

where $A$ is a parameter depending on the actual diameter $d$ of the discs on the calibration plate. The value of $A$ is determined by the least mean square (LMS) method and its evolution as a function of $d$ is presented in Fig. 11. As explained later, the fitted curve in Fig. 11 is useful to correct the effect of the DOF. It has also been verified that the measurement error is weakly affected by the background illumination.

Figures 12 and 13 reveal how the maximum gradient $(G)$ and the maximum intensity $\left(I_{\max }\right)$ are influenced by the distance $z$ from the focal plane. For a given value of $z, G$ and $I_{\max }$ increase with the disc size and reach a plateau. $I_{\max }$

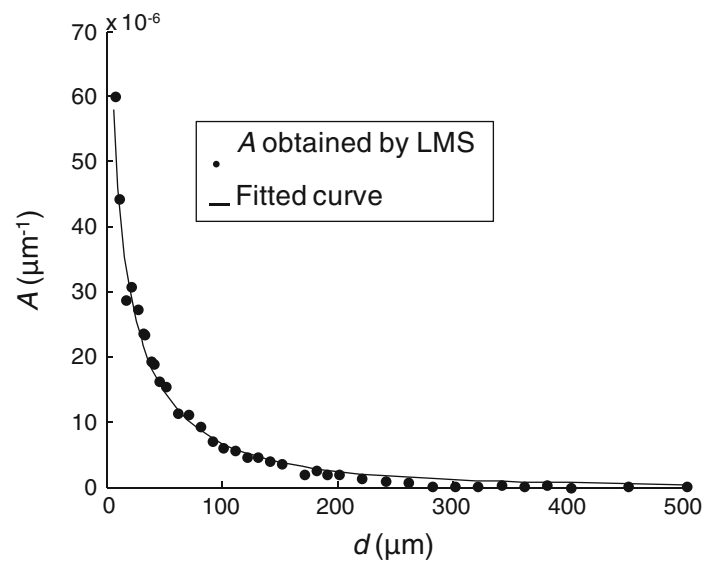

Fig. 11 Evolution of parameter A defined in Eq. (3) as a function of the actual size of the discs on the calibration plate

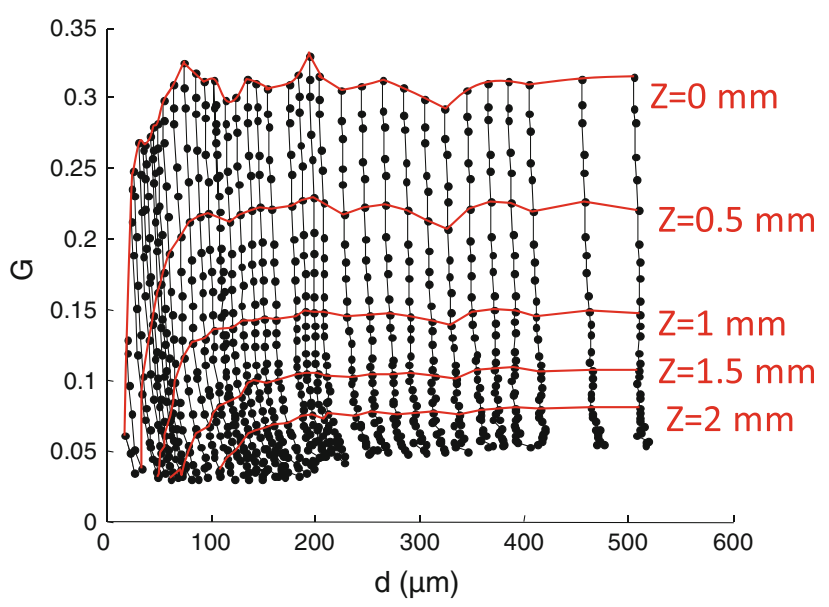

- Measurement values

— Iso-values of disc sizes

- Iso-values of the distance $z$ to the focal plane

Fig. 12 Gradients $G$ at the disc edges in function to the disc sizes for different distances $\mathrm{z}$ from the focal plane ( $d$ is the measured diameter)

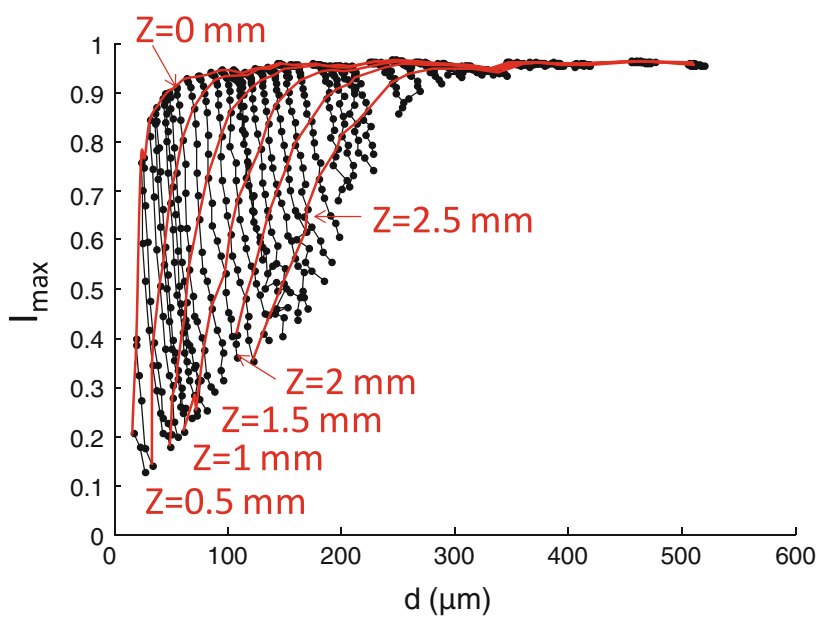

- Measurement values
— Iso-values of disc sizes
— Iso-values of the distance $z$ to the focal plane

Fig. 13 Maximum gray-level intensity as a function of the disc sizes for different distances $z$ from the focal plane $(d$ is the measured diameter)

tends to about 1 whatever the value of $z$, whereas the asymptotic values of $G$ are sensitive to the distance from the focal plane. In order to eliminate droplets out of a prescribed volume, it is clear that a DOF criterion based on the maximum gradient is more relevant for large droplets. This was already stressed by several authors (Koh et al. 2001; Lee and Kim 2004). For sizes inferior to about $150 \mu \mathrm{m}$, 
Fig. 14 Image of the droplet size for the case in Fig. 2a (a diameters based on the equivalent disc area, b diameters averaged by category and track) (a)

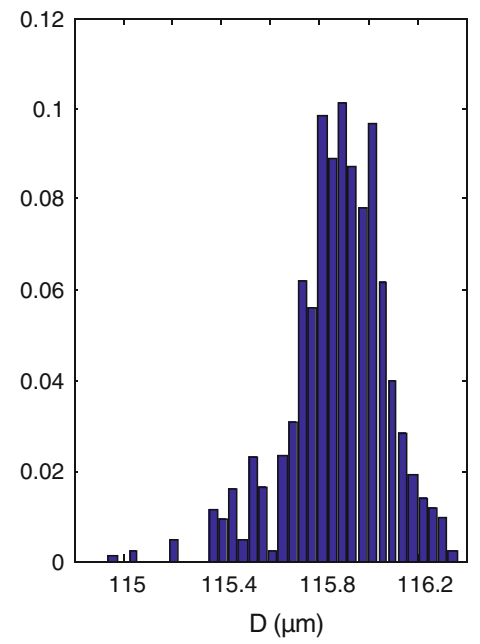

(a)

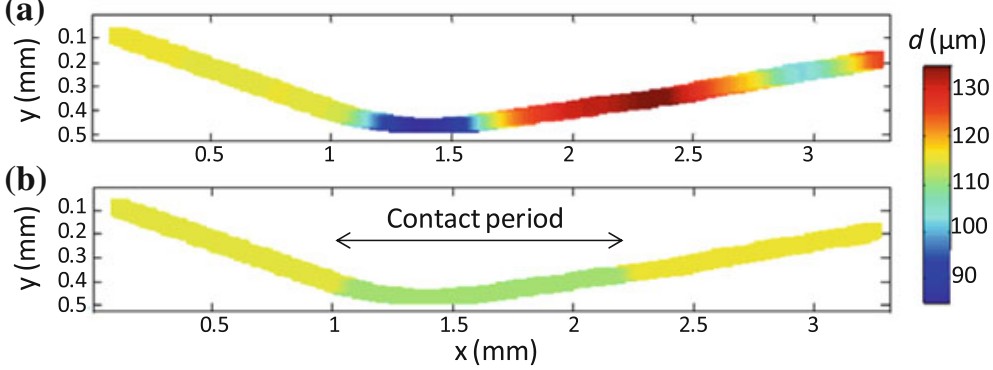

(b)

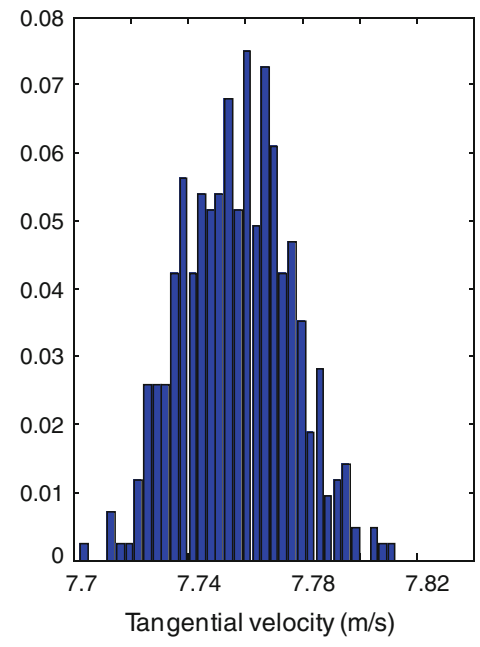

(c)

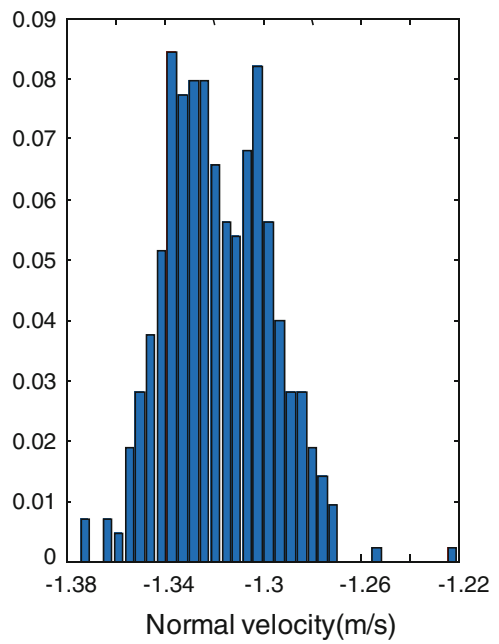

Fig. 15 Size and velocity distributions of the secondary droplets (case of the rebound in Fig. 2 a).a $D_{10}=115.88 \mu \mathrm{m}, \sigma_{D}=0.198 \mu \mathrm{m}$, b $V=7.76 \mathrm{~m} / \mathrm{s}, \sigma_{V}=0.0187 \mathrm{~m} / \mathrm{s}, \mathbf{c} V=-1.32 \mathrm{~m} / \mathrm{s}, \sigma_{V}=0.0213 \mathrm{~m} / \mathrm{s}$

$G$ and $I_{\max }$ exhibit a roughly similar sensitivity to $z$ and can be used therefore as indicators for a DOF criterion.

\section{Results and discussion}

The measurement method described above was used to study the cases of rebound and splashing in Fig. 2.

\subsection{Application to droplet rebounds}

Rebounds are the simplest cases to analyze since all the droplets remain in the focal plane of the camera or very close to it. There is no problem with the effect of the DOF, and tracking droplet trajectories is particularly simple. Size measurement errors are mainly related to the deformation of the droplet after rebound. These errors are partially due to the fact that size measurement is based on the diameter of the disc of equivalent area. Figure 14a shows the space distribution of the droplet size and the deviation induced by the definition of the equivalent diameter can be clearly seen. Errors can be important for the secondary droplets and the droplets in contact with the wall as they are very deformed. In practice, a size is attributed to each track (Lagrangian approach) which allows significantly reducing these errors. Objects are sorted in categories (primary, secondary, droplet in contact with the wall) and the size of each element in a track is replaced by the average size of the track elements belonging to the same category. Figure 14b shows the benefit for measurement accuracy of this averaging method. The same averaging is done later for the splashing. Only the droplets in contact to wall have a diameter slightly above the others. Size and velocity distributions of secondary droplets are presented in Fig. 15. These distributions are relatively narrow and their corresponding standard deviations (about $0.2 \mu \mathrm{m}$ and $0.02 \mathrm{~m} / \mathrm{s}$, respectively) give an idea of the measurement uncertainties. As the droplet velocity and the size of the droplets are measured simultaneously, it is possible to plot the evolution of the droplet deformations as a function of the time spent in contact with the wall (Fig. 16). The residence time, as well as the maximum spreading diameter, can be deduced from this figure. Both parameters are of prime importance for the modeling of the droplet/wall interactions as they are related to the heat transfer rate between the wall and the droplet (Wachters and Westerling 1966). 


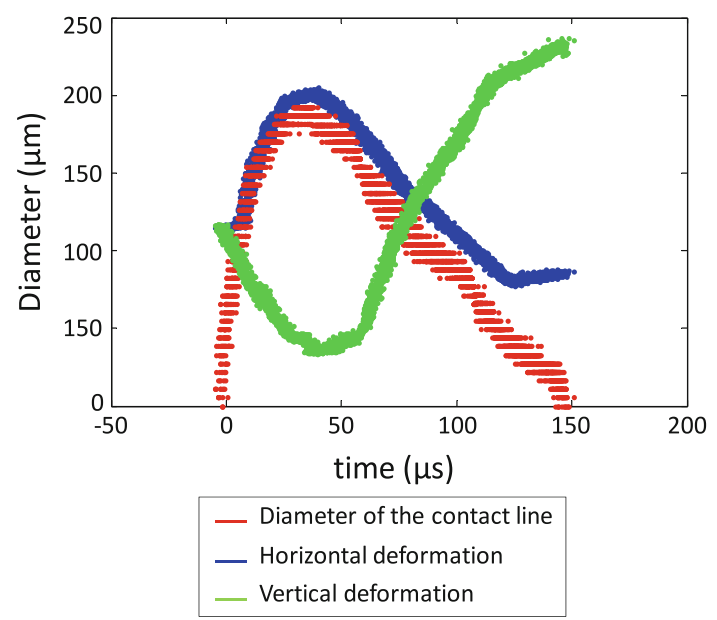

Fig. 16 Deformation of the droplet in contact with the wall (case of the rebound in Fig. 2a)

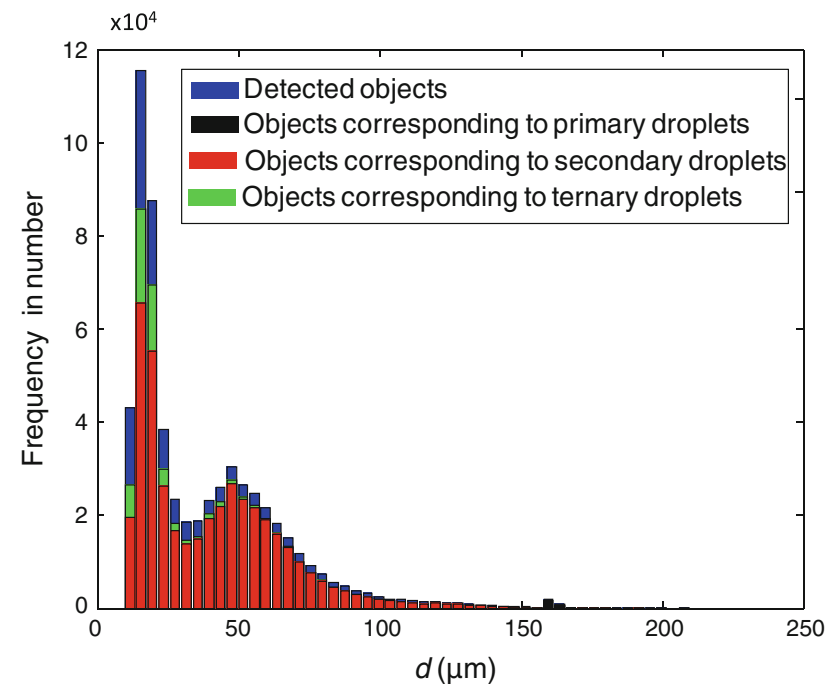

Fig. 17 Size distribution of the detected objects by category in the case of the splashing in Fig. $2 b$

\subsection{Application to droplet splashing}

The analysis of the results is much more complicated in the case of a splashing as special care should be taken to characterize and limit the biases. In order to estimate the performances of the tracking method, the rejection rate (percentage of objects not included in a track) is calculated. It is $19.9 \%$ for the example of Fig. $2 b$ (in comparison, it is almost 0 in the case of the rebound of Fig. 2a and about $10 \%$ for the moderate splashing in Fig. 2c). Figure 17 shows the size distribution of the objects by categories. The size distribution of objects included in a track is very similar to that of all the detected objects. The rejection is slightly more important for the smaller droplets.

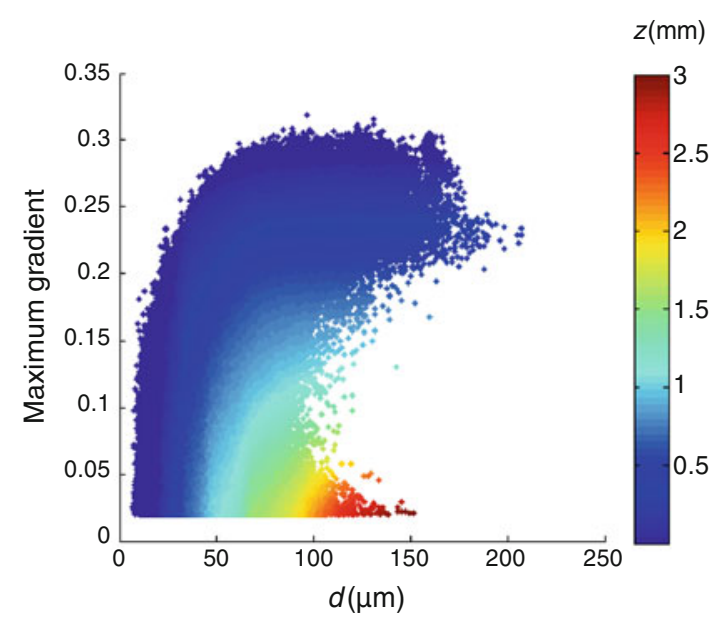

Fig. 18 Maximum gradient versus diameter in the case of the splashing in Fig. 2b (color scale corresponds to the estimated distance $z$ from the focal point)

Droplets having an apparent size smaller than $10-15 \mu \mathrm{m}$ are certainly missing in Fig. 17. The detection limit is thus between 1 and 2 pixels, which is in good agreement with the measurements of the disc sizes of the calibration plate (Fig. 8).

The volume flow rates of primary and secondary droplets have been compared. For the calculations, the volume carried by all the tracks in the category of interest (a track is counted once) is divided by the time period of the acquisition. It appears that the volume of the secondary droplets is about twice that of the primary droplets. If calculations are restricted to the tracks that are crossing a closed frontier around the impact region, the two volume flow rates are found to be rather close, volume carried by the secondary droplets being about $10 \%$ lower than that of the primary droplets. These errors are mainly related to masked droplets and overlapping droplets that are not separated.

The DOF is also a possible source of errors. Figure 18 presents the maximum gradient at the edge of the detected object as a function of their measured sizes. Interpolation based on the data of the calibration plate of Fig. 12 allows evaluating the distance $z$ from the focal plane. The color scale of Fig. 18 is related to the estimated values of $z$. From that figure, it appears that $75 \%$ of the detected objects fall within the interval $0<z<0.5 \mathrm{~mm}$ and thus are weakly affected by size measurement errors induced by DOF (Fig. 10). Only $1.8 \%$ of the detected objects are beyond $z=1.5 \mathrm{~mm}$.

To minimize the effect of DOF, one common method is to limit the thickness of the measurement volume. This has been tested in Fig. 19, where the size distribution is plotted after removing objects having values of $z$ higher than 0.5 or $1 \mathrm{~mm}$. In Fig. 19, the frequencies of the largest secondary 


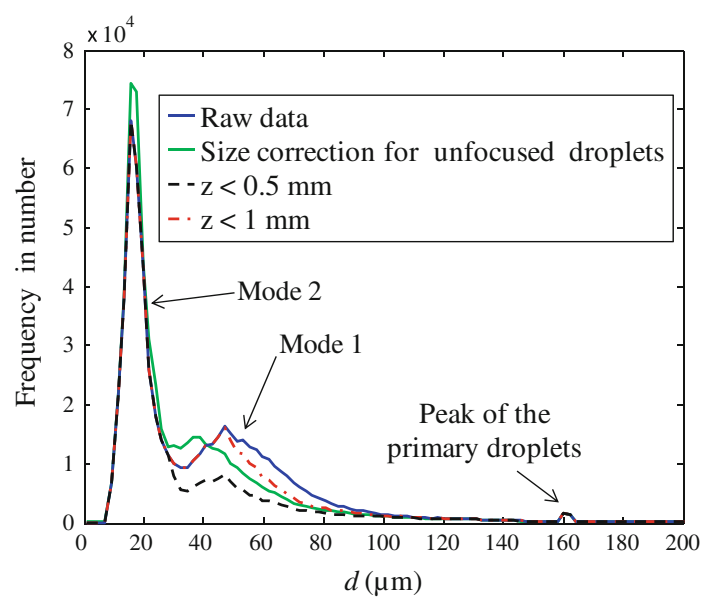

Fig. 19 Influence of the DOF on the size distribution in the case of the splashing in Fig. $2 b$

droplets are the more affected by these removals. This is due to the fact that blurred objects are perceived to be larger than they are actually. For example, a moderately small droplet of $35 \mu \mathrm{m}$ can be detected up to $1.5 \mathrm{~mm}$ from the focal plane (Fig. 9) but its measured size is roughly $80 \mu \mathrm{m}$ at this distance (Fig. 10). This droplet will fall within the same class as a truly focused droplet of $80 \mu \mathrm{m}$ in Fig. 19. In the opposite, droplets that are measured to be small have necessarily a small $\mathrm{z}$ values and thus their actual size is small. Reducing the thickness of the measurement volume can be a perfect approach if it is accompanied by a mapping of the flow field through a sectioning like in tomography. Otherwise, removing blurred objects results in losing information.

As an alternative, an attempt was made to correct the sizes of all the droplets especially the blurred ones. The estimated values of $z$ in Fig. 18 can be used to determine the size overestimation $\operatorname{Err}_{d}$ using Eq. (2). However, the actual droplet size is required to obtain the value of $A$ using Fig. 8. An iterative procedure is thus performed. At each step of the iterations, $d$ in Eq. (2) is replaced by the measured size subtracted by the value of $\operatorname{Err}_{d}$ obtained at the previous step. Three or four iterations are required to converge with a prescribed precision of $0.1 \mu \mathrm{m}$. The size distribution corrected from blurred droplets is plotted in Fig. 19. The second half of it (i.e., the largest secondary droplets) is translated of about $10 \mu \mathrm{m}$ to the left compared to the uncorrected size distribution. To apply the previous correction, it is important that motion blur remains negligible. This can be verified on the basis of the velocity distribution plotted in a log-scale in Fig. 20. Given that the exposure time is fixed at $1 \mu \mathrm{s}$ and the pixel resolution is $7.6 \mu \mathrm{m} / \mathrm{pxl}$, a very small fraction of the emitted droplets have actually a velocity that is high enough to move by more than 1 pixel during the exposure time.

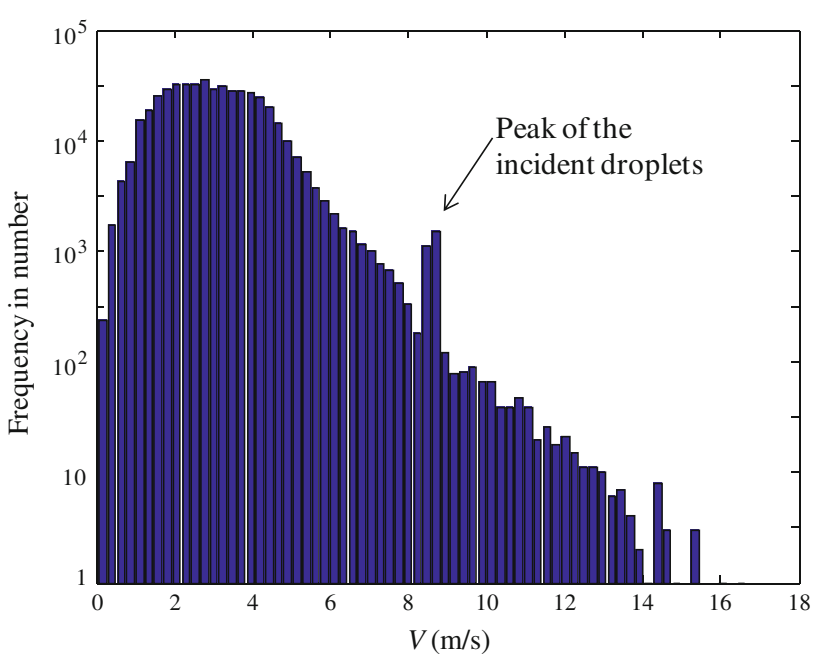

Fig. 20 Velocity distribution of the detected objects

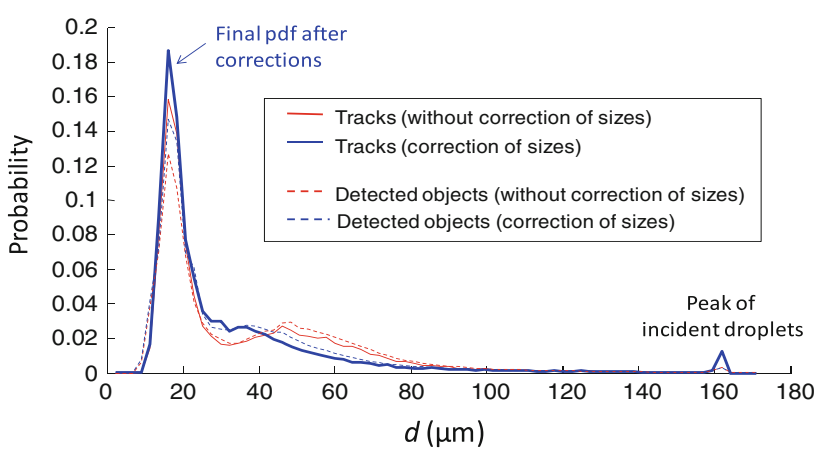

Fig. 21 Size distribution attached to the reconstructed tracks in the case of the splashing in Fig. 2b

As it is, the size distribution in Fig. 19 is still affected by the bias toward the largest droplets that have a larger DOF. For instance, it does not account for the fact that small droplets could be present over a depth greater than their DOF. Even though, this presence seems rather unlikely, since large secondary droplets are very few to be detected beyond $2 \mathrm{~mm}$ (Fig. 18). A correction for the bias stemming from the size dependence of the measurement volume can be proposed. Since the secondary droplets are formed near the central position $(z=0)$, none of them (except those with a size less than 1-2 pixels) are missed by the detection. Their trajectory should be also captured by the tracking program if they are present on a sufficient number of frames (i.e., their velocity along the z-axis is not too large). Using the average size associated with the reconstructed tracks appears rather interesting to calculate the size pdf. Doing so, a droplet would have the same statistical weight whether it is large or small, whether it moves fast or not. This approach is likely to correct the bias toward the slowest droplets that are counted in more frames. Figure 21 shows the size distribution attached to 
the reconstructed tracks. A moderate increase in the number of the smallest droplets can be noticed. As mentioned previously, most of the droplets are located at a distance lower than $1 \mathrm{~mm}$ from the focal plane which could explain certainly why this last correction has a limited effect.

Finally, the impact in Fig. 2c has been also studied. This case is rather close to the transition between the bouncing and the splashing regimes as it corresponds to a Weber number equal to 70 (Wachters and Westerling 1966). The size pdf of the secondary droplets appears to be multimodal and four peaks can be clearly identified in Fig. 22. As presented in Fig. 23, each mode of the pdf can be isolated in order to examine separately the spatial distribution of the corresponding droplets (Fig. 24) and their velocity (Figs. 25, 26). When two modes overlap, the droplets are assigned to the mode that is the most likely (higher frequency in the pdf in Fig. 22).

Figure 24a shows that droplets corresponding to mode 1 are the first created, as they appear at lower $x$ values than the other secondary droplets. As droplets are moving from the left to the right in the frames, the $x$-abscise is roughly correlated with time. Observation of the raw images

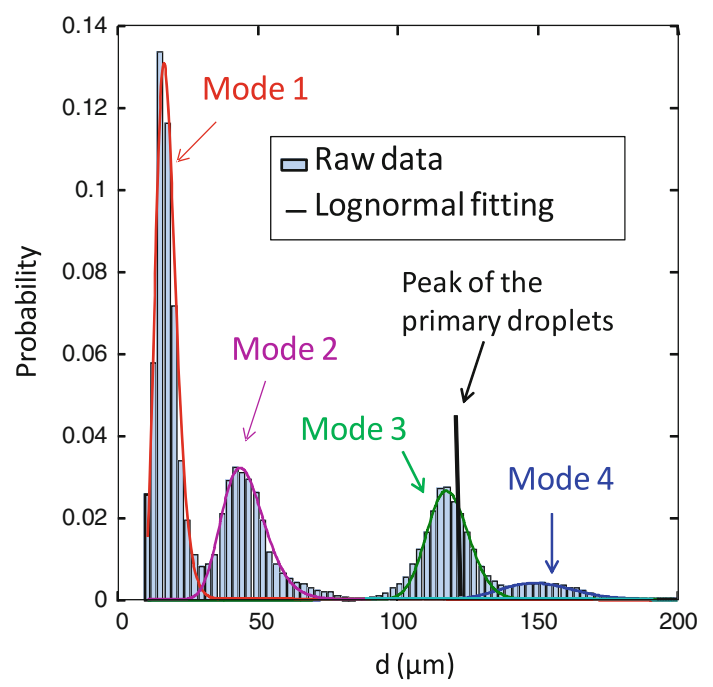

Fig. 22 Size distribution of the secondary droplets in the case in Fig. 2c
(Fig. 2c) reveals that droplets of mode 1 are released at the very beginning of the impact during the spreading of the liquid film. These droplets result from the burst of vapor bubbles produced by the boiling of the liquid film. This process explains their small sizes. Figures $25 \mathrm{~b}$ and $26 \mathrm{~b}$ indicate that they are ejected mostly vertically. Their initial vertical velocity is much higher than that of the other secondary droplets (Fig. 25). As these droplets are very small, they have little momentum and are thus rapidly entrained by larger droplets (Fig. 26b). After the spreading phase, the liquid film is receding like in a rebound (Fig. 16). However, deformation is so strong that breakup occurs shortly after the beginning of the receding phase. This results in a satellite droplet (mode 2) and a main droplet (mode 3). It can be noticed that mode 2 and 3 have roughly the same probability (Fig. 22). It can be also checked that the volume of a primary droplet $\left(d_{10}=119 \mu \mathrm{m}\right)$ is roughly equal to the sum of the volumes of a droplet in mode $2\left(d_{10}=46 \mu \mathrm{m}\right)$ and a droplet in mode $3\left(d_{10}=116 \mu \mathrm{m}\right)$. Satellites droplets have a low normal velocity which explains that they are distributed at small heights from the wall. Mode 4 corresponds to droplets that have a larger size than the primary droplets. The presence of mode 4 can be explained by coalescences of consecutive droplets. Secondary droplets leave the wall with a reduced velocity and thus are spaced more closely than the primary droplets before impact. The short distance between them promotes coalescence between droplets of mode 3. In Fig. 2c, it is also interesting to notice that consecutive primary droplets almost merge during their spreading at the wall surface. This is potentially another route of formation for mode 4 .

\section{Conclusions}

This study highlights the potentials of high-speed shadow imagery for characterizing the size and the velocity of the secondary droplets produced by drop impacts onto heated surfaces. Shadow imagery allows obtaining highly contrasted images that are ideal for detecting the droplet outlines. It offers a way of sizing droplets that are deformed.

Fig. 23 Assignment by modes of the droplets in the image $2 \mathrm{c}$

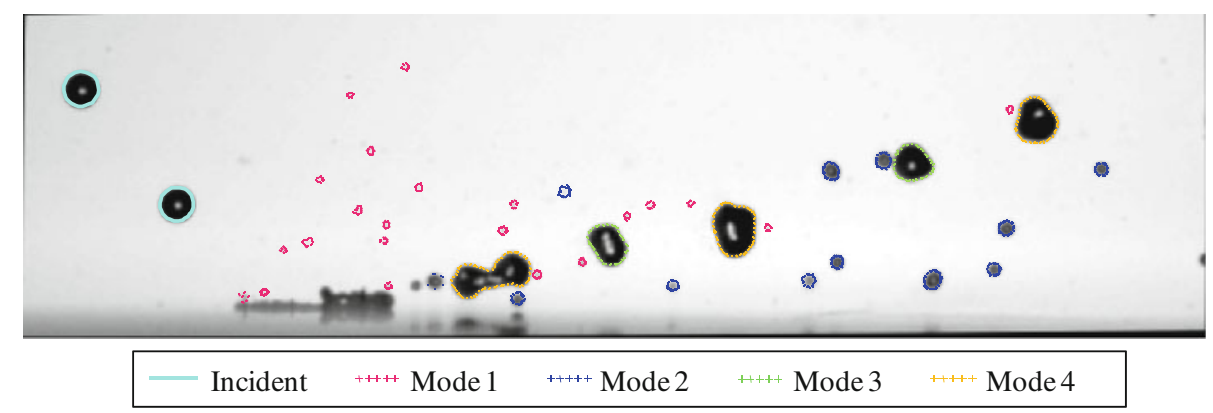



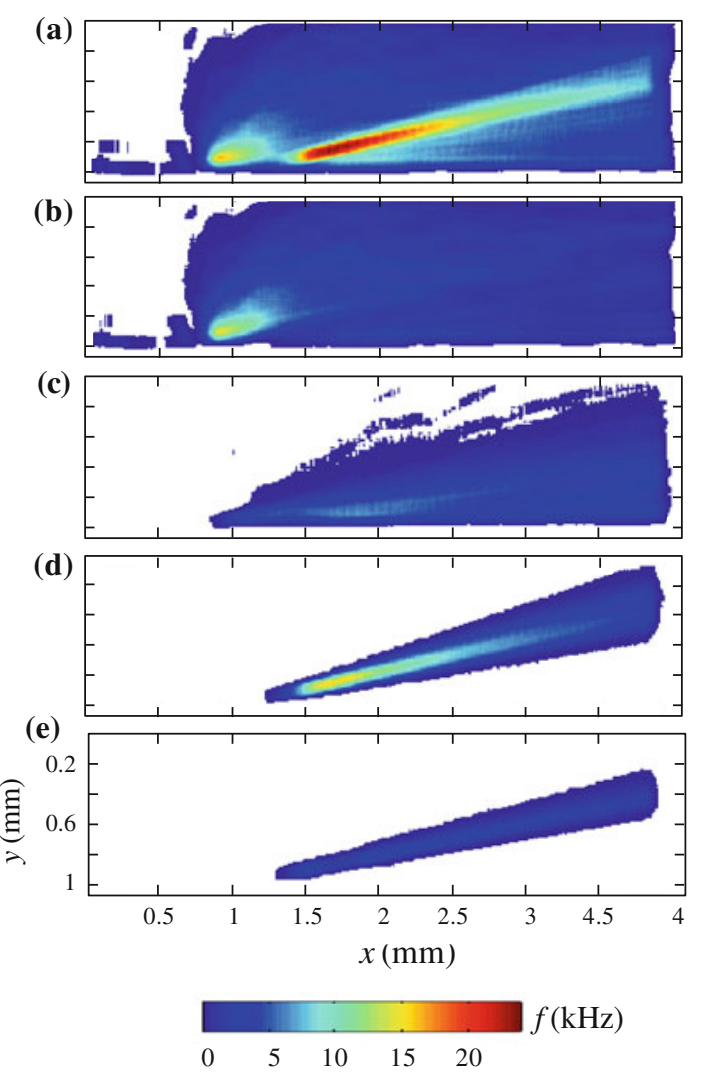

Fig. 24 Spatial distribution of the droplet frequency in $\mathrm{kHz}$ in the case of Fig. 2c (a all the secondary droplets, b mode 1, c mode 2, d mode 3 , e mode 4)

In the presented experiments, the simultaneous presence of small and large droplets makes it difficult to have a fixed threshold level of intensity or gradient for droplet detection. Alternatively, the droplet outline is assimilated to the steepest gradient and it is detected by looking at the zeros of the Laplacian. The implemented image processing implies a Gaussian pre-filtering and a thresholding of the Laplacian to avoid selecting false objects due to noise ripples. Two complementary approaches have been developed to separate overlapping droplets. One is based on the analysis of the shape of the outline; the other relies on the watershed transform.

As high-speed camera is capable of grabbing timeresolved frames at pixel resolution compatible with droplet sizing, a particle tracking algorithm was implemented to determine the droplet velocity in the two dimensions of the images. Being adapted from multiple hypothesis methods, this algorithm is particularly robust. When combined to size measurements, tracking offers some additional features. For example, it allows correcting the bias of the size distribution toward the slowest droplets when droplets have significantly different velocities. It allows minimizing the sizing errors due to droplet deformation by averaging the size of the track elements. The direction of the droplet

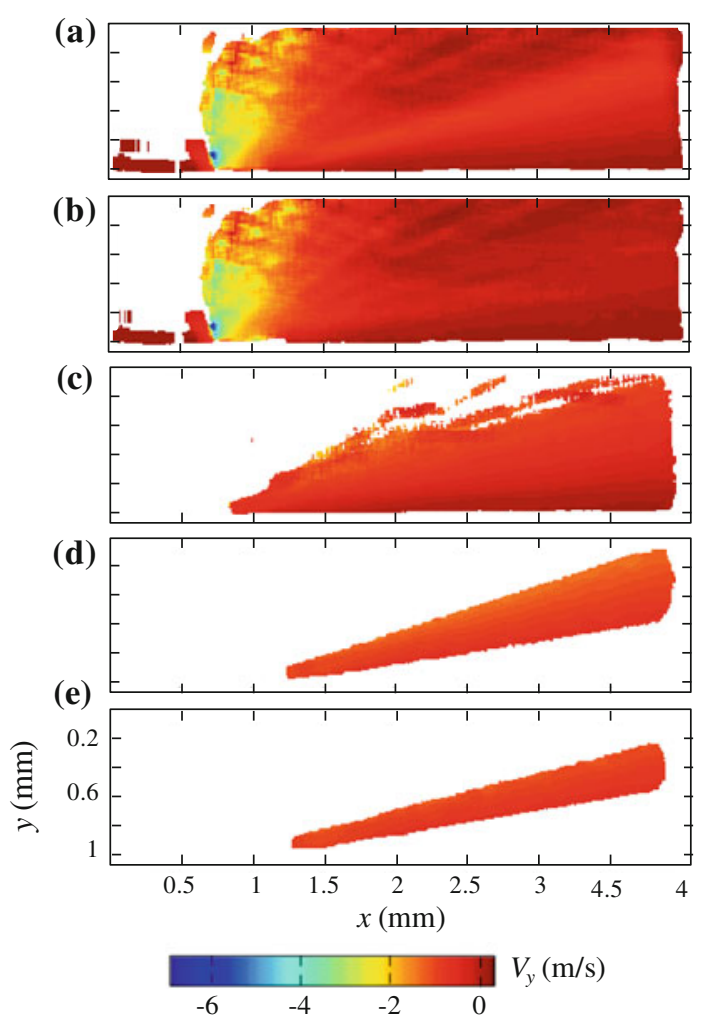

Fig. 25 Spatial distribution of the droplet vertical velocity in $\mathrm{m} / \mathrm{s}$ in the case of Fig. 2c (a all the secondary droplets, $\mathbf{b}$ mode 1, $\mathbf{c}$ mode 2, d mode 3 , e mode 4)

velocity makes it easy to distinguish secondary droplets from primary ones.

A calibration plate dotted with discs of different sizes is required to assess the accuracy of the size measurements. This plate is also useful to characterize the depth of field and the resulting sizing errors. The calibration plate is moved back and forth along the camera axis, which reveals also how the gradient at an object outline is changing with its distance from the focal plane. Based on these observations, the maximum gradient appears to be a reliable indicator to evaluate droplet distances from the focal plane. It can therefore be used as a criterion to eliminate droplets that are out of a predefined measurement thickness. A correction of the size of the blurred droplets is also possible based on this indicator.

In addition, the application of the technique to rebounds allows determining the residence time and monitoring the time evolution of the spreading diameter. For splashing, as different modes of re-atomization can be identified, it is possible to characterize separately the size and velocity distributions of the associated droplets.

Although developed for characterizing drop impacts, the measurement method is expected to be relevant to other multiphase flows including sprays and bubbly flows. The 

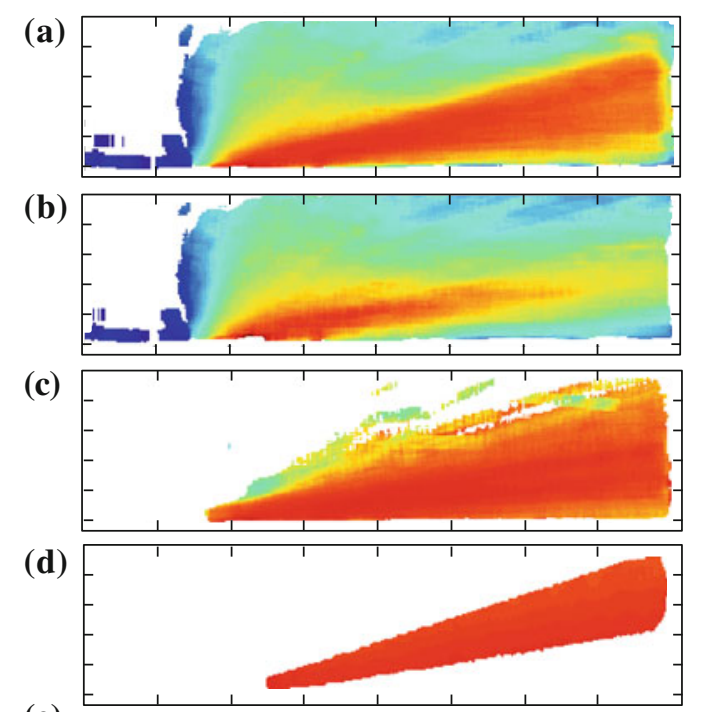

(e)
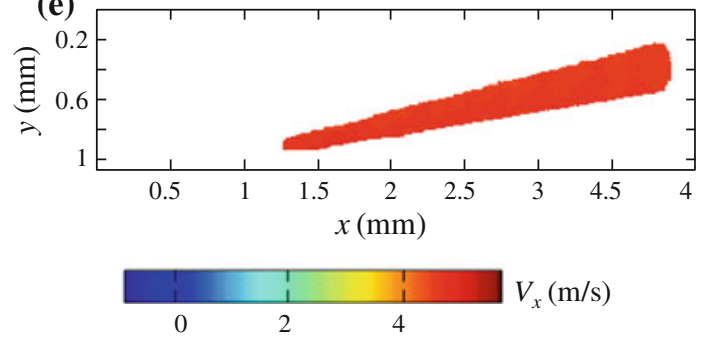

Fig. 26 Spatial distribution of the droplet horizontal velocity in $\mathrm{m} / \mathrm{s}$ in the case of Fig. 2c (a all the secondary droplets, $\mathbf{b}$ mode 1, $\mathbf{c}$ mode 2 , d mode 3 , e mode 4)

developed image-based technique can be seen as an alternative or a complement to the well-established phase Doppler analysis (PDA). It has the ability to describe the shape of liquid particles that are not fully atomized or relaxed, while an high-speed visualization offers the possibility to reconstruct full trajectories of the particles and thus to have a Lagrangian description of the flow. However, among the remaining limits of the technique is the problem of overlapping particles and the unavoidable compromise between time and space resolution when rapid phenomena like the breakup are of interest. An extension of this work would be to compare measurements by these two techniques to assess the performance of the developed image approach and investigate its areas of applicability.

Acknowledgments This work has been supported by the French National Agency (ANR) in the frame of the research program IDHEAS (ANR-NT09 432160).

\section{References}

Acharya T, Ray AK (2005) Image processing: principles and applications. Wiley-Interscience. ISBN: 978-0-471-71998-4

Bernardin JD, Mudawar I (1997) Film boiling heat transfer of droplet streams and sprays. Int J Heat Mass Transf 40:2579-2593
Beucher S, Meyer F (1993) The morphological approach to segmentation: the watershed transformation. In: Dougherty ER (ed) Mathematical morphology in image processing. The morphological approach to segmentation: the watershed transform, chap 12, pp 433-481

Biance AL, Clanet C, Quere D (2003) Leidenfrost drops. Phys Fluids 15:1632-1637. doi:10.1063/1.1572161

Blaisot J, Yon J (2005) Droplet size and morphology characterization for dense sprays by image processing: application to the Diesel spray. Exp Fluids 39:977-994. doi:10.1007/s00348-005-0026-4

Bongiovanni C, Chevaillier JP, Fabre J (1997) Sizing of bubbles by incoherent imaging: defocus bias. Exp Fluids 23:209-216. doi: $10.1007 /$ s003480050104

Castanet G, Lienart T, Lemoine F (2009) Dynamics and temperature of droplets impacting onto a heated wall. Int J Heat Mass Transf 52:670-679. doi:10.1016/j.ijheatmasstransfer.2008.07.024

Chenouard N, Bloch I, Olivo-Marin J-C (2009) Multiple hypothesis tracking in cluttered condition. In: International conference on image processing, Cairo, Egypt

Cossali GE, Marengo M, Santini M (2005) Secondary atomisation produced by single drop vertical impacts onto heated surfaces. Exp Thermal Fluid Sci 29:937-946

Damaschke N, Gouesbet G, Gréhan G, Mignon H, Tropea C (1998) Response of PDA systems to non-spherical droplets. Appl Opt 37:1752-1761

Dunand P, Castanet G, Lemoine F (2012a) A two-color planar LIF technique to map the temperature of droplets impinging onto a heated wall. Exp Fluids 52:843-856

Dunand P, Gradeck M, Castanet G, Lemoine F, Maillet D (2012) Energy balance in case of single or multiple droplets impinging onto a hot slab in the Leidenfrost regime. In: ICLASS 2012, 12th Triennial international conference on liquid atomization and spray systems, Heidelberg, Germany

Fantini E, Tognotti L, Tonazzini A (1990) Drop size distribution in sprays by image processing. Comput Chem Eng 14:1201-1211

Fdida N, Blaisot JB, Floch A, Dechaume D (2010) Drop-size measurement techniques applied to gasoline sprays. At Sprays 20:141-162

Hay KJ, Liu ZC, Hanratty TJ (1998) A backlighted imaging technique for particle size measurements in two-phase flows. Exp Fluids 25:226-232. doi:10.1007/s003480050225

Kashdan JT, Shrimpton JS, Whybrew A (2003) Two-phase flow characterization by automated digital image analysis. Part 1: fundamental principles and calibration of the technique. Part Part Syst Charact 20:387-397. doi:10.1002/ppsc.200300897

Kim KS, Kim SS (1994) Drop sizing and depth-of-field correction in TV imaging. At Sprays 4:65-78

Kim JY, Chu JH, Lee SY (1999) Improvement of pattern recognition algorithm for drop size measurement. At Sprays 9:313-329

Koh KU, Kim JY, Lee SY (2001) Determination of in-focus criteria and depth of field in image processing of spray particles. At Sprays 11:317-333

Lebrun D, Touil CE, Özkul C (1996) Methods for the deconvolution of defocused-image pairs recorded separately on two CCD cameras: application to particle sizing. Appl Opt 35:6375-6381

Lecuona A, Sosa PA, Rodriguez PA, Zequeira RI (2000) Volumetric characterization of dispersed two-phase flows by digital image analysis. Meas Sci Technol 11:1152-1161

Lee S, Kim Y (2004) Sizing of spray particles using image processing technique. J Mech Sci Technol 18:879-894. doi:10.1007/bf029 90860

Malot H, Blaisot J-B (2000) Droplet size distribution and sphericity measurements of low-density sprays through image analysis. Part Part Syst Charact 17:146-158. doi:10.1002/1521-4117 (200012)17:4<146:aid-ppsc146>3.0.co;2-4

Moita AS, Moreira ALN (2012) Scaling the effects of surface topography in the secondary atomization resulting from droplet/ wall interactions. Exp Fluids 52:679-695 
Moreira ALN, Moita AS, Cossali E, Marengo M, Santini M (2007) Secondary atomization of water and isooctane drops impinging on tilted heated surfaces. Exp Fluids 43:297-313

Müller A, Dullenkopf K, Bauer H-J (2008) Application of an extended particle tracking method to analyze droplet wall interaction. In: 14th international symposium on applications of laser techniques to fluid mechanics, Lisbon, Portugal

Nishino K, Kato H, Torii K (2000) Stereo imaging for simultaneous measurement of size and velocity of particles in dispersed twophase flow. Meas Sci Technol 11:633-645

Oberdier LM (1984) An instrumentation system to automate the analysis of fuel-spray images using computer vision. ASTM 848:123-136
Reid DB (1979) An algorithm for tracking multiple targets. IEEE Trans Automat Contr 24(6):843-854

Richter B, Dullenkopf K, Bauer HJ (2005) Investigation of secondary droplet characteristics produced by an isooctane drop chain impact onto a heated piston surface. Exp Fluids 39:351-363. doi: 10.1007/s00348-005-1018-0

Van der Veen RCA, Tran T, Lohse D, Sun C (2012) Direct measurements of air layer profiles under impacting droplets using high-speed color interferometry. Phys Rev E 85:6. doi: 10.1103/PhysRevE.85.026315

Wachters LHJ, Westerling NAJ (1966) The heat transfer from a hot wall to impinging water drops in the spheroidal state. Chem Eng Sci 21:1047-1056 